\title{
Intra- and early postoperative complications after standard cataract surgery or femtosecond laser-assisted cataract surgery combined with pars plana vitrectomy in cases of retinal diseases
}

\author{
Fritz H. Hengerer ${ }^{*}$, Gerd U Auffarth ${ }^{2}$ and Ina Conrad-Hengerer ${ }^{2}$ \\ ${ }^{1}$ Eye Hospital at Bürgerhospital Frankfurt - Goethe-University Frankfurt, Germany; IVCRC.net - Research Network in Ophthalmology, Heidelberg, Germany \\ ${ }^{2}$ Department of Ophthalmology, Ruprecht-Karls-University of Heidelberg, Germany; IVCRC.net - Research Network in Ophthalmology, Heidelberg, Germany
}

\begin{abstract}
Background and objective: To assess intra- and postoperative anterior chamber stability and IOL position during sutureless 23 -gauge pars plana vitrectomy (23g PPV) with gas endotamponade after standard or Femtosecond laser-assisted cataract surgery.

Study Design/Materials and methods: All patients had to undergo pars plana vitrectomy, because of a retinal disease, in combination with cataract surgery and volunteered for the study. The trial received ethical committee approval (Heidelberg, Germany) and all aspects of the Helsinki Declaration were observed. After randomization, 65 eyes were treated by laser-assisted cataract surgery and the other 66 eyes had phacoemulsification using pulsed ultrasound energy and intraocular lens implantation. In both groups cataract surgery was followed by a $23 \mathrm{~g}$ PPV with gas or silicone oil endotamponade. All intraoperative and early postoperative complications were documented. The effective phaco time and further additional surgical steps to achieve a stable IOL-position or anterior chamber were analyzed. IOL-centration and changes in anterior chamber were assessed using slit-beam microscopy of the operation microscope. Furthermore, the $360^{\circ}$ overlap of capsulorrhexis/capsulotomy and IOL optic was assessed.

Results: The study group with 65 femtosecond laser-assisted cataract surgeries (FLACS group) showed a significant lower EPT in comparison to the standard cataract group. All patients in the FLACS group showed a $360^{\circ}$ overlap of capsulotomy and IOL optic after gas or silicone oil tamponade, but in the standard group 45 of 66 eyes showed $360^{\circ}$ overlap of capsulorrhexis and IOL optic after endotamponade as well as a flattening of the anterior chamber in the center part. 10 eyes needed repositioning of the IOL (1 FLACS group, 9 standard group) at the end of surgery due to dislocation of the IOL.
\end{abstract}

Conclusions: The Femtosecond Laser capsulotomy guarantees a perfect $360^{\circ}$ overlap of the capsulotomy with the IOL. This should protect from any complications associated with PPV and intraoperative filling of the vitreous body with an endotamponade.

\section{Precis}

Cataract surgery is one of the most successful procedures in medicine. However, the combination of PPV with standard cataract surgery often shows intraoperative and early post-operative complications due to instable anterior chamber situations.

More important in case of combined surgery with PPV is that the capsulotomy carried out by a femtosecond laser guarantees a perfect $360^{\circ}$ overlap of the capsulotomy with the IOL even in eyes with lack of red reflex or shallow anterior chamber. This should protect from any complications associated with an intraoperative filling of the vitreous body with expanding gas/air fill.

Our study showed that femtosecond laser-assisted capsulotomy and lens fragmentation can be performed safely in a one-step approach performing $23 \mathrm{~g}$ PPV resulting in perfectly centered capsulotomies and individually selected suitable IOLs as well as excellent surgical postOP outcomes with less intra- and early postoperative complications, respectively.

\section{Introduction}

In eyes with retinal disorders and the need of surgical intervention by pars plana vitrectomy performing a standard cataract surgery can be a great challenge. Absence of red reflex is one of the reasons for intraoperative capsular complications and a safe IOL implantation cannot be achieved in all cases. A stable and well-centered IOL is the base for the following pars plana vitrectomy to have full access to the vitreous cavity without touching the natural lens. Furthermore, the IOL is used as a diaphragm and stabilizes the anterior segment during the time of gas or silicone oil endotamponade in the vitreous cavity.

${ }^{*}$ Correspondence to: Fritz $\mathrm{H}$. Hengerer, MD, PhD, FEBO, Eye Hospital at Bürgerhospital Frankfurt - Goethe-University Frankfurt, Germany, Nibelungenallee 37-41, 60318 Frankfurt am Main, Germany, E-mail: f.hengerer@ buergerhospital-ffm.de

Key words: femtovitrectomy, FLACS, phacoemulsification, sutureless vitrectomy, complications

Received: April 01, 2021; Accepted: April 16, 2021; Published: April 22, 2021 
Hengerer FH (2021) Intra- and early postoperative complications after standard cataract surgery or femtosecond laser-assisted cataract surgery combined with pars plana vitrectomy in cases of retinal diseases

Many efforts can be undertaken to enhance visualization of the anterior lens capsule to create a continuous curvilinear capsulorrhexis. Using dyes like trypan blue or other colours like in white cataracts are not helpful in a non-mature lens and projection of a circle by the microscope also does not allow a better view towards the anterior lens capsule.

Since more than ten years femtosecondlaser-assisted cataract surgery (FLACS) has been established as a new and safe ophthalmic surgical procedure in standard and difficult cataract surgery. By the help of the laser system intraoperative OCT imaging allows to create a threedimensional model of the whole anterior segment with all margins of the natural lens regardless of a red reflex. After precise analysis of the structures the laser allows to achieve a circular capsulotomy centered to the pupil or aligned with the scanned capsular bag, respectively. The natural lens can be precut into small cubicles or segments to save ultrasound energy during phacoemulsification and lens aspiration protecting the cornea and intraocular structures.

In our study we combined cataract surgery with pars plana vitrectomy in cases of retinal diseases and compared standard manual cataract surgery with femtosecondlaser-assisted cataract surgery. The advantage of combined surgery is the complete removal of vitreous, access to peripheral retina, full indentation possible, no lens touch, no tamponade-induced cataract and most of all a "single surgery" versus "secondary surgical approach". Furthermore, the aspect of covering the surgical cost for treating such eyes is favorable.

The aim of our prospective randomized clinical trial was to assess the intra- and postoperative anterior chamber stability and IOL position during sutureless 23-gauge pars plana vitrectomy (23g PPV) with gas or silicone oil endotamponade after standard cataract surgery or Femtosecond laser-assisted cataract surgery.

\section{Patients/Materials and methods}

The study was performed at our University Eye Hospital. All patients had to undergo pars plana vitrectomy, because of retinal disease, in combination with cataract surgery and volunteered for the study. The trial received ethical committee approval and all aspects of the Helsinki Declaration were observed.
The femtosecond laser procedure with Catalys Precision Laser System (Johnson \& Johnson Vision, New Brunswick, California, USA) was used to perform capsulotomy as well as lens fragmentation prior to cataract surgery. For phacoemulsification or lens aspiration after femtosecond laser procedure as well as for standard cataract surgery and 23g-pars plana vitrectomy we used Stellaris Elite (Bausch \& Lomb, Aliso Viejo, California, USA). All patients received an IOL-implantation with a preloaded single-piece IOL (TECNIS PCB00, Johnson \& Johnson Vision, New Brunswick, California, USA).

Patients were randomized into two groups. All patients received a number and each number was allocated by computer generated sequence. After randomization 65 eyes were treated by femtosecond laser-assisted cataract surgery (FLACS group) and the other 66 eyes underwent phacoemulsification (standard group) using manual capsulorhexis and pulsed ultrasound energy, all eyes received intraocular lens implantation (Table 1). Both options of cataract surgeries were followed by a $23 \mathrm{~g}$ PPV with gas or silicone oil endotamponade according to the retinal pathology. All intra- and postoperative complications were documented. The used effective phaco time and further additional surgical steps to regain a stable IOL-position or anterior chamber were analyzed. IOL-centration and lens tilt were assessed using slitlamp examination. Furthermore a $360^{\circ}$ overlap of capsulorrhexis/ capsulotomy and IOL optic was observed in all cases.

Our study design was a randomized, prospective controlled clinical trial. Inclusion criteria were retinal diseases necessary to be treated by vitrectomy as well as senile cataract. Exclusion criteria were small pupils less than $6 \mathrm{~mm}$ after pharmacological mydriasis with phenylephrine and tropicamid eye drops administered 3 times, corneal opacification, mature brown cataracts, lax zonules and pseudoexfoliation syndrome.

All patients were prepared according to our standard laser cataract surgery protocol by application of eye drops with a non-steroidal antiphlogistic agent Nepafenac 1mg (Nevanac, Alcon, USA) three times one hour before surgery as well as topical 0.5\% Mydriaticum (Tropicamid, Stulln Pharma, Germany) and 5.0\% Neo-Synephrine (phenylepherine, Ursapharm, Germany) instilled 15 minutes three times within one hour prior to surgery. Randomization took place before surgery opening an envelope for each patient and FLACS group

Table 1. Demographics and Retinal Pathologies. Preoperative axial length was $23.55 \mathrm{~mm} \pm 1.06(21.83-27.05 \mathrm{~mm})$ in the LCS group and $23.55 \mathrm{~mm} \pm 1.07(21.88-27.13 \mathrm{~mm})$ in the standard group

\begin{tabular}{|c|c|c|}
\hline & FLACS* group & Standard ${ }^{* *}$ group \\
\hline Number (of eyes) & 65 & 66 \\
\hline Age (years) & $\begin{array}{c}66.1+/-9.4 \\
\text { median: } 68.0\end{array}$ & $\begin{array}{l}63.0+/-10.6 \\
\text { median: } 64.5\end{array}$ \\
\hline Gender (male / female) & $31 / 34$ & $32 / 34$ \\
\hline Axial Length (mm) & $\begin{array}{c}23.65 \mathrm{~mm} \pm 1.12 \\
\text { median: } 23.72\end{array}$ & $\begin{array}{c}23.58 \mathrm{~mm} \pm 1.07 \\
\text { median: } 23.67\end{array}$ \\
\hline $\begin{array}{l}\text { Pentacam Nucleus Staging } \\
\text { Pentacam Nucleus Density } 1(\%) \\
\text { Pentacam Nucleus Density } 2(\%)\end{array}$ & $\begin{array}{c}1.09+/-0.80 \\
\text { (median: } 1.00 \text { ) } \\
10.92+/-1.98 \\
\text { (median: } 10.65 \text { ) } \\
10.70+/-3.27 \\
\text { (median: } 10.20 \text { ) }\end{array}$ & $\begin{array}{l}1.06+/-0.92 \\
\text { (median: } 1.00 \text { ) } \\
10.81+/-2.34 \\
\text { (median: } 10.60 \text { ) } \\
10.45+/-1.88 \\
\text { (median: } 10.00 \text { ) }\end{array}$ \\
\hline Macular pucker / hole & $24 / 12$ & $22 / 13$ \\
\hline Vitreous hemorrhage & 13 & 12 \\
\hline Retinal detachment & 16 & 18 \\
\hline
\end{tabular}

* FLACS- femtosecond laser-assisted cataract surgery

** Standard - conventional phacoemulsification 
Hengerer FH (2021) Intra- and early postoperative complications after standard cataract surgery or femtosecond laser-assisted cataract surgery combined with pars plana vitrectomy in cases of retinal diseases

was placed after topical anesthesia on the laser bed whereas standard group was placed directly on the operation bed.

The femtosecond laser-assisted cataract surgeries group (FLACS group) underwent femtosecond laser-assisted capsulotomy of $5 \mathrm{~mm}$ in diameter centered to the optical axis. Lens fragmentation/softening ( $350 \mu \mathrm{m}$; Quadrants; Catalys Laser System, Figure 1) was performed thereafter. All pupils in the FLACS group dilated more than $6 \mathrm{~mm}$ and no change to capsulotomy diameter had been necessary. After undocking from the laser system the operation was continued under the operation microscope.

All further steps were performed similar in both groups according to our standard cataract protocol in our clinical setting. Therefore, we decided not to use the femtosecond-laser for the corneal incisions. Two paracenteses were placed at 10 and 2 o'clock with a metal keratome (2-gauche, Alcon, Fort Worth, Texas, USA) and the main incision was placed at $12 o^{\prime}$ clock with an angled slit knife of $2.4 \mathrm{~mm}$ width (Alcon, Fort Worth, Texas, USA). The anterior chamber was filled with Healon GV (Johnson \& Johnson Vision, New Brunswick, California, USA) as ocular viscosurgical device (OVD) and the capsulotomy disc was pulled out with forceps. Hydrodissection was followed by phacoaspiration of the fragmented nucleus and with bimanual irrigation and aspiration remaining cortex was removed from the capsule. Implantation of a preloaded single piece aspheric monofocal IOL was performed after filling the capsular bag with Healon GV. The IOL was centered and all OVD in the anterior chamber and the capsular bag was removed even behind the IOL by bimanual irrigation and aspiration. All corneal incisions were meticulously hydrated to achieve a watertight situation with a stable anterior segment. The depth of the anterior chamber was assessed by slit beam microscopy of the operation microscope in the pupil center with regard to the corneal thickness in the center part. A minimum of four corneal thickness was graded as steep. Then three $23 \mathrm{~g}$ trocars (Bausch \& Lomb, Aliso Viejo, California, USA) for pars plana vitrectomy were placed temporal inferior and at 10 and $2 o^{\circ}$ clock in line with the paracenteses.

The standard group underwent manual capsulorrhexis aiming for at least $5 \mathrm{~mm}$ in diameter using an Utrata forceps (Geuder, Heidelberg, Germany) followed by phacoemulsification of the crystalline lens (Figure 2). After this, both patient groups received sutureless three-port 23-gauge pars plana vitrectomy and gas/silicone oil endotamponade.

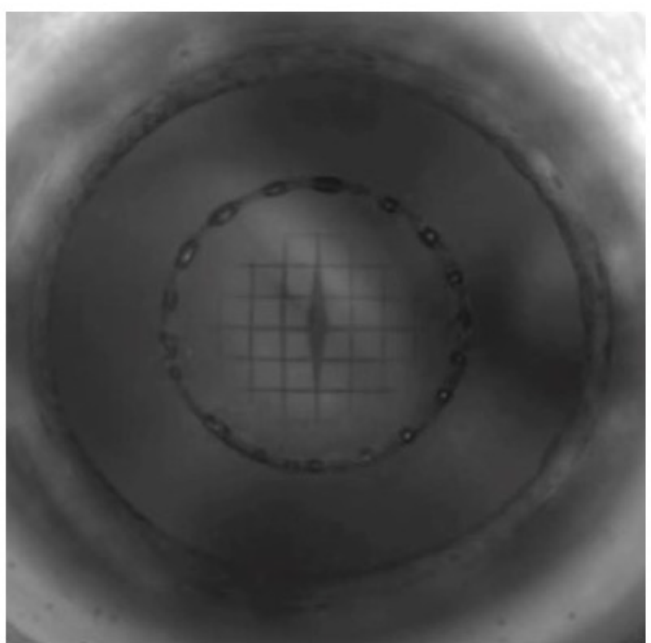

Figure 1. Femtosecond laser-assisted capsulotomy and lens fragmentation / softening (350 $\mu \mathrm{m}$; Quadrants; Catalys Precision Laser System)

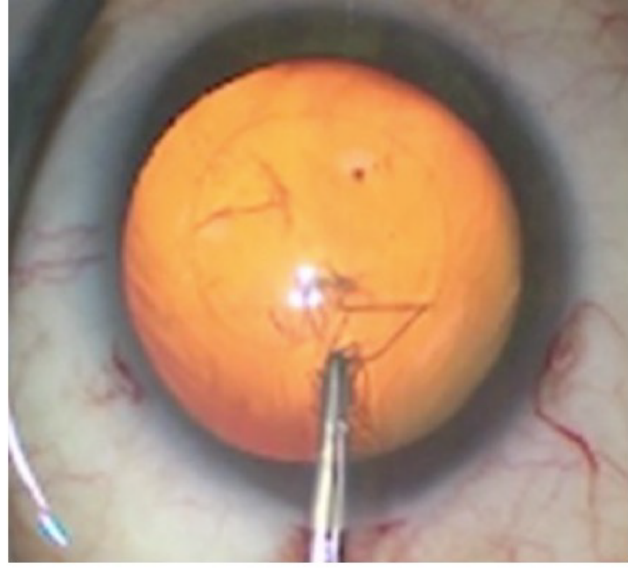

Figure 2. Manual capsulorrhexis of the anterior lens capsule

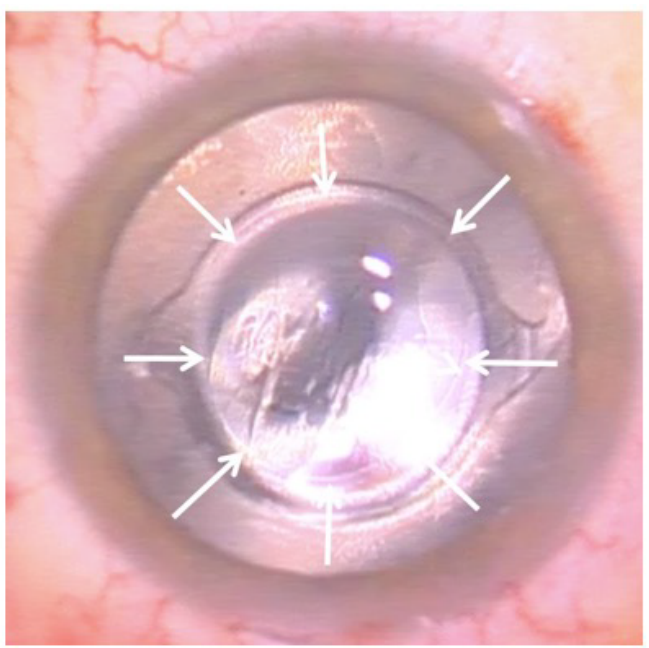

Figure 3. Femtosecond laser-assisted capsulotomy with gas tamponade (capsulotomy-IOL optic overlap of $360^{\circ}$ )

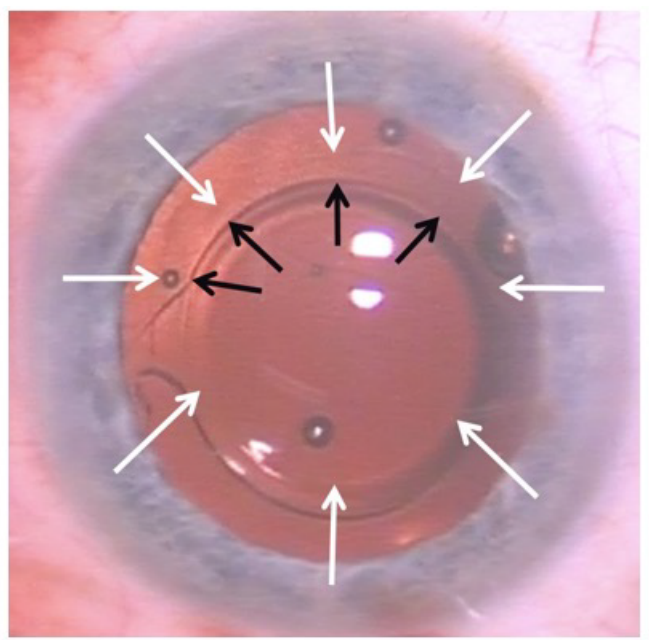

Figure 4. Manual capsulorrhexis with silicon oil tamponade (capsulorrhexis-IOL optic overlap of approximately $240^{\circ}$ ) 
Hengerer FH (2021) Intra- and early postoperative complications after standard cataract surgery or femtosecond laser-assisted cataract surgery combined with pars plana vitrectomy in cases of retinal diseases

Anterior vitrectomy with removal of vitreous behind the capsular bag was followed by posterior vitrectomy using BIOM5 wide angle view (Oculus, Wetzlar, Germany) for visualization. A standard handheld shielded illumination lightpipe was used both for endoillumination as well as indentation of the globe to visualize peripheral vitreous remnants at pars plana during vitreous shaving diaphanoscopically. Brilliant peel (Geuder, Heidelberg, Germany) was used in all cases to stain epiretinal membranes. No triamcinolone has been used at all. According to the underlying retinal pathology either 20\% SF6 gas tamponade (Fluoron, Neu-Ulm, Germany) or silicone oil 5000 centistoke (Fluoron, NeuUlm, Germany) had been used after fluid-air exchange.

The IOL-position was checked before vitrectomy and after fluid-gas or fluid-silicone oil exchange by the surgeon and another ophthalmological surgeon and graded independently. All surgeries were videotaped in case of necessary further analyses.

After one week the IOL-position was assessed with slit-lamp biomicroscopy (Haag-Streit BQ, Köniz, Switzerland) after applying mydriatic eye drops (tropicamid and phenylephrine) and graded by two independent physicians.

A stable IOL-position was defined and graded as follows:

- IOL in capsular bag

- Capsulotomy/capsulorrhexis-IOL optic overlap of $360^{\circ}$

- anterior chamber depth more than 4 times center corneal thickness height from endothelium to IOL

An instable IOL-position was defined and graded as follows:

- IOL out of capsular bag in part or total

- iris capture of the IOL

- decentration of IOL optics of more than $1 \mathrm{~mm}$ with or without Capsulotomy/capsulorrhexis-IOL optic overlap of $360^{\circ}$

- anterior chamber depth less than 4 times center corneal thickness height from endothelium to IOL

Anterior chamber depth was graded as follows:

- steep (more than 4 times center corneal thickness height from endothelium to IOL)

- flat (less than 4 times center corneal thickness height from endothelium to IOL)

- lost (less than 1 time center corneal thickness height from endothelium to IOL)

In cases of an instable IOL position during vitrectomy or after fluidair exchange, the IOL was repositioned immediately using bimanual irrigation/aspiration or in combination with a blunt phaco spatula. No further ocular viscosurgical device had been used.

\section{Statistical analysis}

All descriptive statistical analysis was conducted using SPSS Version 22.0 (SPSS Inc, Chicago, Illinois, USA). We used a t-test to compare the sample means. A P-value less than 0.05 was considered statistically significant.

\section{Results}

The demographic data and cataract density (as presented in table 1) showed no significant differences. In both groups the IOL could be implanted primarily without capsule complications and centered according to the pupil. Assessment of the effective phaco time (EPT; $100 \%$ ultrasound) was significantly different and it resulted in $0.21 \pm$ 0.12 seconds ( $\mathrm{s}$ ) for eyes in the FLACS group versus $1.41 \mathrm{~s} \pm 1.14$ in the standard group.

Before vitrectomy all cases presented a stable IOL-position as defined above and anterior chamber depths were steep. Two eyes (one in each group) received silicone oil endotamponade according to severe proliferative vitreoretinopathy. In all other cases gas tamponade $(20 \%$ SF6 gas tamponade) was sufficient.

Capsulotomy/capsulorrhexis-IOL optic overlap of $360^{\circ}$ was achieved in $100 \%$ of the femtosecond laser group, in $62 \%$ of the eyes undergoing manual capsulorrhexis (FLACS group (65/65), standard group (41/66); significant difference).

Anterior chamber depth was rated as steep in $98 \%$ of the FLACS group (64/65) and as flat in one eye, and as steep in $89 \%$ of the eyes treated in the standard group (59/66), as flat in five eyes and was lost in one eye, respectively.

A stable IOL-position at the end of operation showed $98 \%$ in the FLACS group (64/65) and one IOL decentered more than $1 \mathrm{~mm}$ and had to be repositioned.

In $86 \%$ of the standard group (58/66) the IOL was stable whereas in 3 cases the IOL was out of the capsular bag and decentered in 5 additional cases with the need of an immediate surgical approach. IOL repositioning was necessary in $2 \%$ of the FLACS group (1/65), in $12 \%$ of the standard group (8/66) (statistically significant difference).

One week postoperative the IOL-position was stable in $100 \%$ treated by FLACS (65/65), 84\% in the standard group (56/66) (statistically significant difference). IOL-repositioning was necessary in a secondary approach in no eye of the FLACS group (0/66), in 5\% of the standard group due to iris capture (3/66, two eyes filled with gas, one eye filled with silicone oil).

\section{Discussion}

Compared to manual cataract surgery, which has been established for more than 40 years, the femtosecond laser-assisted surgical procedure represents a further surgical development. A lot of publications about FLACS discuss several aspects such as reduction to zero ultrasound energy, reduction of postoperative inflammation in the anterior segment and endothelial cell stability over time using a femtosecond laser [1-6]. Furthermore, results focus on the circularity and precision of the capsulotomy highlighting its accuracy compared with the procedure done manually $[7,8]$.

Vote et al. [9] states that over time the clinical indications for FLACS have become quite narrow. Now in less than $5 \%$ of all cases, including those with very mature cataract or those with significant corneal endothelial compromise, it is used. For the majority of patients in their opinion FLACS does not provide either sufficient clinical benefit or cost-effectiveness to warrant this use [10]. Still the proof of an improved effective lens position after FLACS is missing, although some papers have supported reduced IOL tilt and aberrations [11,12]. Alio et al. [13] and O'Brart et al. [14] summarized, FLACS seems to be beneficial in some eyes, but that despite its advanced technology, the advantages might not be clear in routine cases and that FLACS could not be considered as cost-effective. 
Hengerer FH (2021) Intra- and early postoperative complications after standard cataract surgery or femtosecond laser-assisted cataract surgery combined with pars plana vitrectomy in cases of retinal diseases

The efficacy and safety of FLACS combined with vitrectomy have been published as well $[15,16]$. In our randomized, prospective controlled clinical trial we focused on CCC-IOL-overlap and intra-/ postoperative decentration of the IOL after pars plana vitrectomy using gas or silicone endotamponade. All FLACS study group eyes had a stable IOL-position caused by $360^{\circ}$ CCC-IOL-overlap and one eye had to undergo IOL repositioning at the end of surgery. At one week visit no IOL decentration or iris capture was appearant. Jung et al. [15] and Gómez-Resa et al. [16] have reported similar observations.

In our standard group 8 eyes had to undergo a secondary intervention at the end of surgery to achieve a well-positioned IOL. All eyes received a gas endotamponade. After one week in two eyes with gas endotamponade and silicone oil fill in one eye caused IOL-decentration and IOL-reposition got necessary.

This surgery can be challenging because pressure from the vitreous cavity caused by tamponade lead to immediate flattening of the anterior chamber when opening the paracenteses. Using irrigation can be helpful but fluid will pass through zonules towards the vitreous cavity and after IOL-reposition a second fluid-air exchange can be necessary. In cases with silicone oil tamponade re-opening of the anterior chamber can be crucial as silicone oil can egress from posterior. Therefore, we recommend the use of OVD for stabilizing the anterior chamber to avoid silicone oil slippage or underfill due to aqueous fluid moving posteriorly during the repositioning maneuver.

Another aspect has to be discussed according to additional costs by using a femtosecond Lasersystem for cataract surgery. In refractive cataract surgery all additional costs were directly charged to the patient asking for this technology. As this is generally accepted as an ambulatory procedure, all in-house treatments like combined pars plana vitrectomy or cataract surgery belong to the DRG system. In this system a certain amount of payback exists for all treatment categories and devices used in a surgical case regardless additional costs for more expensive methods like femtosecond Lasers, respectively. This has to be taken into consideration when the benefits pointed out in our study have to be compared with the lower costs for a manual procedure and the need for additional surgical interventions. Other surgeons may use a Zepto capsulotomy device (Centricity Vision, Carlsbad, California, USA) to create a CCC but limitations here are flattened anterior chamber or small pupils which interfere with this approach. Furthermore, a larger main incision is needed to use this device as we have in our standard regimen.

Use of rhexis calipers may support some help in cataract surgery cases with red reflex in presence but most of the retinal cases suffer from lack of red reflex. Even additional dyes like trypan blue do not enhance visualization here.

Other aspects like small incision cataract surgery beyond $1.8 \mathrm{~mm}$ can be safely used with Stellaris system for combined procedures. Limitations are only hydrophilic lenses so far fit through this incision size with limited optical diameters less than $6 \mathrm{~mm}$. We decided for our study to use the same preloaded single-piece aspheric hydrophobic IOL as we use for our standard cataract surgery in our hospital.

Our study has some limitations according to the intraoperative assessment of the anterior chamber depth. We had to use a slitbeam from the operation microscope due to non- availability of an intraoperative Optical Coherence Tomography (OCT) which could analyze the depth of the anterior chamber precisely. This may be used in future studies to be more accurate.
Achieving a $360^{\circ}$ overlap with the IOL helps to avoid these complications and is one of the benefits from FLACS-surgery in these cases. Furthermore, use of intraoperative OCT- imaging provided by the femtosecond laser system helps to avoid staining of the lens capsule in patients with lack of red reflex caused by vitreo-retinal pathologies like vitreous hemorrhage or retinal detachment. These study results could show evidence for femtosecond laser-assisted cataract surgery in eyes with retinal pathologies being superior to manual cataract removal. This can open the field for more clinical applications focusing on less complications and secondary surgeries in eyes suffering from retinal diseases in contrast to FLACS as a refractive procedure, respectively $[17,18]$.

\section{Acknowledgments}

This paper has not been presented at any meeting yet.

The authors have no financial interest in the products mentioned.

This study was supported by an unrestricted educational grant from Johnson \& Johnson Vision.

\section{References}

1. Donaldson KE, Braga-Mele R, Cabot F, Davidson R, Dhaliwal DK, et al. (2013) ASCRS refractive cataract surgery subcommittee. J Cataract Refract Surg 39: 17531763.

2. Nagy Z, Takacs A, Filkorn T, Sarayba M (2009) Initial clinical evaluation of an intraocular femtosecond laser in cataract surgery. J Refract Surg 25: 1053-1060. [Crossref]

3. Kim P, Sutton GL, Rootman DS (2011) Applications of the femtosecond laser in corneal refractive surgery. Curr Opin Ophthalmol 22: 238-244.

4. Trikha S, Turnbull AMJ, Morris RJ, Anderson DF, Hossain P (2013) The journey to femtosecond laser-assisted cataract surgery: new beginnings or a false dawn. Eye (Lond) 27: 461-473. [Crossref]

5. Abell RG, Allen PL, Vote BJ (2013) Anterior chamber flare after femtosecond laserassisted cataract surgery. J Cataract Refract Surg 39: 1321-1326. [Crossref]

6. Abouzeid H, Ferrini W (2014) Femtosecond-laser assisted cataract surgery: a review. Acta Ophthalmol 92: 597-603. [Crossref]

7. Reddy KP, Kandulla J, Auffarth GU (2013) Effectiveness and safety of femtosecond laser-assisted lens fragmentation and anterior capsulotomy versus the manual technique in cataract surgery. J Cataract Refract Surg 39: 1297-1306. [Crossref]

8. Abouzeid H, Ferrini W (2014) Femtosecond-laser assisted cataract surgery: a review. Acta Ophthalmol 92: 597-603. [Crossref]

9. Hooshmand J, Vote BJ (2017) Femtosecond laser-assisted cataract surgery, technology, outcome, future directions and modern applications. Asia Pac J Ophthalmol (Phila) 6 : 393-400. [Crossref]

10. Schweitzer C, Brezin A, Cochener B, Monnet D, Germain C, et al. (2020) Femtosecond laser-assisted versus phacoemulsification cataract surgery (FEMCAT): a multicentre participant-masked randomised superiority and cost-effectiveness trial. Lancet 395: 212-224. [Crossref]

11. Alió JL, Abdou AA, Puente AA, Zato MA, Nagyet Z (2014) Femtosecond laser cataract surgery: updates on technologies and outcomes. J Refrac Surg 30: 420-427. [Crossref]

12. Mihaltz K, Knorz MC, Alió JL, Takács AI, Kránitz K, et al. (2011) Internal aberrations and optical quality after femtosecond laser anterior capsulotomy in cataract surgery. $J$ Refrac Surg 27: 711-716. [Crossref]

13. Kanclerz P, Alio JL (2020) The benefits and drawbacks of femtosecond laser-assisted cataract surgery. Eur J Ophthalmol 1120672120922448.

14. Roberts HW, Day AC, O`Brart DPs (2020) Femtosecond laser-assisted cataract surgery: A review. Eur J Ophthalmol 30: 417-429.

15. Jung Y, Kim IN, Yoon J, Lee JY, Kim KH, et al. (2013) Intracameral illuminatorassisted advanced cataract surgery combined with 23 -gauge vitrectomy in eyes with poor red reflex. J Cataract Refract Surg 39: 845-850. [Crossref] 
Hengerer FH (2021) Intra- and early postoperative complications after standard cataract surgery or femtosecond laser-assisted cataract surgery combined with pars plana vitrectomy in cases of retinal diseases

16. Gómez-Resa M, Nieto I, Corcóstegui B (2014) Combined 23-gauge vitrectomy and femtosecond laser-assisted cataract surgery. Ophthalmic Res 52: 141-146. [Crossref]

17. Conrad-Hengerer I, Al Sheikh M, Hengerer FH, Schultz T, Dick HB, et al. (2015) Comparison of visual recovery and refractive stability between femtosecond laserassisted cataract surgery and standard phacoemulsification: six-month follow-up. $J$ Cataract Refract Surg 41: 1356-1364. [Crossref]
18. Alió JL, Abdou AA, Soria F, Javaloy J, Fernández-Buenaga R, et al. (2013) Femtosecond laser cataract incision morphology and corneal higher-order aberration analysis. J Refract Surg 29: 590-595. [Crossref]

Copyright: (C2021 Hengerer FH. This is an open-access article distributed under the terms of the Creative Commons Attribution License, which permits unrestricted use, distribution, and reproduction in any medium, provided the original author and source are credited. 